\title{
Inhibition of EGFR signaling abrogates smooth muscle proliferation resulting from sustained distension of the urinary bladder
}

\author{
Carlos R Estrada ${ }^{1,2}$, Rosalyn M Adam ${ }^{1,2}$, Samuel H Eaton ${ }^{1}$, Darius J Bägli ${ }^{3}$ and \\ Michael R Freeman ${ }^{1,2,4}$ \\ ${ }^{1}$ Urological Diseases Research Center, Department of Urology, Children's Hospital Boston, Boston, MA, USA; \\ ${ }^{2}$ Department of Surgery, Harvard Medical School, Boston, MA, USA; ${ }^{3}$ Division of Urology, The Hospital for \\ Sick Children, University of Toronto, Toronto, ON, Canada and ${ }^{4}$ Department of Biological Chemistry and \\ Molecular Pharmacology, Harvard Medical School, Boston, MA, USA
}

\begin{abstract}
Urinary bladder outlet obstruction results in sustained stretch of the detrusor muscle and can lead to pathological smooth muscle hyperplasia and hypertrophy. The epidermal growth factor receptor (EGFR) is a cognate receptor for mitogens implicated in bladder hyperplasia/hypertrophy. Here, we investigated the potential for modulation of this pathway by pharmacologic targeting with a clinically available EGFR antagonist using an organ culture model of bladder stretch injury as a test system. Urinary bladders from adult female rats were distended in vivo with medium containing the EGFR inhibitor ZD1839 (gefitinib, Iressa). The bladders were excised and incubated in ex vivo organ culture for 4-24 h. EGFR phosphorylation, DNA proliferation, and the extent of apoptosis in the cultured tissues were assessed. To verify that the smooth muscle cells (SMC) are a target of the EGFR inhibitor, primary culture human and rat bladder SMC were subjected to cyclic mechanical stretch in vitro in the presence of ZD1839. Levels of phosphorylated EGFR were significantly increased in the detrusor muscle with $12 \mathrm{~h}$ of stretch in the organ cultures. This activation coincided with a subsequent 23 -fold increase in DNA synthesis and a 30-fold decrease in apoptosis in the muscle compartment at $24 \mathrm{~h}$. In the presence of ZD1839, DNA synthesis was reduced to basal levels without an increase in the rate of apoptosis under ex vivo conditions. Mechanical stretch of bladder SMC in vitro resulted in a significant increase in DNA synthesis, which was completely abrogated by treatment with ZD1839 but not by AG825, an inhibitor of the related receptor, ErbB2. Our results indicate that the EGFR pathway is a physiologically relevant signaling mechanism in hypertrophic bladder disease resulting from mechanical distension and may be amenable to pharmacologic intervention.
\end{abstract}

Laboratory Investigation (2006) 86, 1293-1302. doi:10.1038/labinvest.3700483; published online 16 October 2006

Keywords: bladder; EGFR/ErbB1; mechanotransduction; organ culture; smooth muscle; stretch

Urinary bladder outlet obstruction can result from a variety of anatomical and/or functional abnormalities of the genitourinary system. The opposing physiologic roles of the bladder, prolonged storage of urine under low pressure and acute contraction during urination, are tightly regulated and coordinated such that they occur cooperatively, with the normal bladder emptying completely with voiding. Obstruction of the bladder outlet generates in-

Correspondence: Dr MR Freeman, PhD, Urological Diseases Research Center, Department of Urology, Children's Hospital Boston, 300 Longwood Avenue, John F Enders Research Laboratories, Suite 1161, Boston, MA 02115, USA.

E-mail: michael.freeman@childrens.harvard.edu

Received 8 August 2006; revised 7 September 2006; accepted 8 September 2006; published online 16 October 2006 creased contractile forces during the contraction phase, and also results in incomplete emptying. This condition elicits chronic overdistention (sustained stretch of the tissue) and triggers pathologic remodeling of the bladder wall. Prolonged bladder outlet obstruction results in a fibroproliferative reaction characterized by smooth muscle cell (SMC) hyperplasia/hypertrophy and extracellular matrix deposition. ${ }^{1,2}$ The role of sustained stretch as a discrete stimulus is thought to represent a major contributing factor to the tissue changes seen with obstruction. ${ }^{1,3}$ The ability of a mechanical stimulus to trigger cell growth has been observed in several systems, including vascular SMC, ${ }^{4-6}$ cardiac myocytes, ${ }^{7,8}$ and epithelial cells. ${ }^{9-12}$

Growth factors and other signaling proteins regulate diverse cellular functions, including 
responses to changing physiological conditions. Adaptation to sustained stretch may initially be protective-that is, expansion of the smooth muscle compartment as an adaptive response to augment bladder emptying-but over time such changes can become deleterious. Cell growth responses, such as those observed in SMC hyperplasia and hypertrophy, are partly the result of the action of soluble mitogens. ${ }^{13}$ The downstream targets of these factors provide potential regulatable targets for therapies aimed at modulating aberrant cellular processes.

Growth regulatory proteins whose expression is affected by mechanical forces have been implicated in the response of SMC to tension and pressure. ${ }^{14-18}$ The gene encoding heparin-binding epidermal growth factor-like growth factor (HB-EGF) was identified as stretch- and pressure-responsive in bladder SMC. ${ }^{15,17}$ HB-EGF signals principally through the transmembrane receptor tyrosine kinase (RTK) epidermal growth factor receptor (EGFR, ErbB1), which activates a number of well known signal transduction pathways. ${ }^{19}$ Stretch of bladder SMC in vitro leads to increased DNA synthesis as well as rapid activation of multiple signaling cascades. ${ }^{20}$ Studies on HB-EGF in the urinary tract have suggested a role for the activated EGFR in compensatory bladder hyperplasia and hypertrophy. Some of the evidence for this hypothesis is the following: (1) bladder SMC is a site of HB-EGF synthesis; ${ }^{21,22}$ (2) HB-EGF expression is stretchresponsive in bladder SMC in vitro and in vivo; ${ }^{15,22,23}$ (3) HB-EGF is a bladder SMC mitogen $;^{21}$ and (4) bladder SMC express the EGFR. ${ }^{22}$ In addition, the EGFR has been implicated in stretchinduced growth and proliferation of other hollow organs and structures such as the cardiovascular system, ${ }^{5-7,24-27}$ lung tissue,${ }^{11}$ renal tubules,${ }^{9}$ and the gastrointestinal tract. ${ }^{28}$

In this study, we tested whether (1) controlled distension of the intact urinary bladder in ex vivo organ culture, and of primary culture bladder SMC in vitro, results in EGFR activation and (2) whether pharmacologic blockade of this receptor, using a well-characterized inhibitor, alters the cellular response to the pathologic stimulus. Our results show that downstream effects of the sustained stretch stimulus were attenuated by the pharmacologic blockage regimen, suggesting that the EGFR plays an important role in stretch-induced urinary bladder SMC hyperplasia and hypertrophy. Moreover, such antagonists may have therapeutic benefit in the context of bladder outlet obstruction as a means to protect the tissue from decompensation.

\section{Methods}

All animal experiments were performed and human tissues obtained with approval from the Institutional Animal Care and Use Committee and
Institutional Review Board, respectively, at Children's Hospital Boston.

\section{Ex Vivo Model of Bladder Overdistention}

An ex vivo model of bladder stretch injury was used as previously described. ${ }^{23}$ Briefly, 30-day-old-female Wistar rats were anesthetized with isoflurane inhalation. The bladder was catheterized and drained via a 20-gauge intravenous catheter. A low midline incision was made to expose the bladder, and the ureters were ligated and divided. The bladder neck was isolated and surrounded by a $4-0$ silk suture. The bladder was then stretched to $40 \mathrm{~cm}$ water pressure using a gravity manometer with medium optimized for maintenance of the urothelium (modified keratinocyte growth medium (KGM) supplemented with $2 \%$ fetal bovine serum (FBS)). ${ }^{29}$ The bladder neck suture was then tightened, the catheter removed, and the bladder excised and placed in Dulbecco's modification of Eagle's medium (DMEM) supplemented with $10 \%$ FBS. Bladders were maintained in culture for $4,12,18$, and $24 \mathrm{~h}$ in a humidified $5 \% \mathrm{CO}_{2} / 95 \%$ air atmosphere incubator. As a control, a nonstretched bladder for each time point was harvested and incubated in the identical fashion as the stretched bladders. Four bladders were employed for each time point and each condition (nonstretched and stretched).

\section{In Vitro Cyclic-Stretch Relaxation of Bladder Smooth Muscle Cells}

Primary culture human and rat bladder SMC were obtained as described previously, ${ }^{15,30}$ and maintained in DMEM/10\% FBS, or medium 199 (M199)/20\% FBS, respectively. Cells were routinely cultured at $37^{\circ} \mathrm{C}, 5 \% \mathrm{CO}_{2} / 95 \%$ air in a humidified incubator. Cells were subjected to cyclic-stretch relaxation and ${ }^{3} \mathrm{H}$-thymidine incorporation was assessed essentially as described. ${ }^{15}$ Briefly, cells were serum-depleted for $24 \mathrm{~h}$ and then stretched for $12 \mathrm{~h}, 18 \mathrm{~h}$, and $24 \mathrm{~h}(20 \%$ elongation, $0.1 \mathrm{~Hz})$. For the final $8 \mathrm{~h}$ of stretch, $0.5 \mu \mathrm{Ci} /$ well ${ }^{3} \mathrm{H}$-thymidine was added, and incorporation of radioactivity into acidprecipitable material was measured by scintillation counting. Nonstretched cells that were seeded and incubated in parallel served as controls. In selected wells, cells were incubated with the EGFR-specific tyrosine kinase inhibitor (TKI) ZD1839 $(10 \mu \mathrm{M})$ or the ErbB2-specific inhibitor AG825 (Calbiochem, San Diego, CA, USA) $(0.35 \mu \mathrm{M})$ for $30 \mathrm{~min}$ before initiation of stretch.

\section{Preparation of Whole-Bladder Tissue Lysates}

Tissue from replicate bladders at each time point was combined and placed in $0.5 \mathrm{ml}$ protein extraction buffer (T-Per, Pierce Biotechnology, Rockford, 
IL, USA) containing protease inhibitors (CompleteMini, Roche Applied Science, Indianapolis, IN, USA) on ice for $30 \mathrm{~min}$. Following incubation, the bladder tissue was homogenized (Polytron homogenizer) and centrifuged at 14000 r.p.m. for $5 \mathrm{~min}$. The supernatant was collected and protein concentrations determined using the MicroBCA protein assay reagent (Pierce, Rockford, IL, USA). Lysates were resolved by sodium dodecyl sulfate-polyacrylamide gel electrophoresis (SDS-PAGE) and transferred to nitrocellulose membrane. Equal protein loading was confirmed with Ponceau S staining. Immunoblotting was performed using antibodies against total EGFR (Santa Cruz Biotechnology, Santa Cruz, CA, USA) and phosphorylated EGFR (Tyr-1068, Cell Signaling Technology Inc., Beverly, MA, USA). For enhanced detection of phosphorylated species, immunoprecipitation was performed with antibody against total EGFR. Immunoprecipitates were collected on protein A/G beads, washed extensively in lysis buffer and resolved by SDS-PAGE before immunoblotting with antiphosphotyrosine antibody (Cell Signaling Technology). Isotype IgG served as a negative control. For all immunoblots neonatal rat kidney epithelial cells (NRK-52E) treated with or without 100ng/ml recombinant epidermal growth factor (rEGF, R\&D Systems, Minneapolis, MN, USA) served as a positive control. Semiquantitation of protein expression was determined using image analysis (ImageJ, NIH).

\section{Immunohistochemical Analyses}

Bladder tissue was fixed with $10 \%$ neutral buffered formalin at room temperature for $8 \mathrm{~h}$, washed with phosphate buffered saline (PBS), and dehydrated with ethanol gradient washes. Tissue was then paraffin embedded and $8 \mu \mathrm{m}$ sections were obtained using a microtome. The sections were mounted on glass slides and baked for $30 \mathrm{~min}$ at $70^{\circ} \mathrm{C}$. Sections were deparaffinized and rehydrated using xylene and gradient ethanol washes. Endogenous peroxidase activity was neutralized by incubation in $3 \%$ hydrogen peroxide for $15 \mathrm{~min}$. Sections were washed with either PBS or Tris-buffered saline (TBS) depending on antibody and manufacturer's recommendations. Antibodies included anti-EGFR (Santa Cruz), antiphospho-EGFR (Tyr 1068, Cell Signaling), anti-ErbB2 (Upstate Cell Signaling Solutions, Waltham, MA, USA), antiphospho-erbB2 (Cell Signaling), anti-ErbB3 (Santa Cruz), and anti-ErbB4 (Cell Signaling). All sections were incubated in primary antibody overnight at $4^{\circ} \mathrm{C}$. Appropriate biotinylated secondary antibodies were utilized and antigen-antibody complexes visualized with the avidin-biotin complex (ABC) method (Vector Laboratories, Burlingame, CA, USA). All experiments included negative controls consisting of sections incubated with secondary antibody only. Immunohistochemical staining was examined under brightfield microscopy (Zeiss Axioplan, Carl Zeiss, Thornwood, NY, USA). Stain intensity was quantitated using image analysis (Image J, NIH). Hematoxylin and eosin and Masson's trichrome staining revealed minimal changes in bladder tissue architecture in response to distention, with both the urothelium and SMC compartments remaining intact for at least $24 \mathrm{~h}$ in culture (data not shown).

\section{5-Bromo2'-deoxyuridine and TUNEL Staining}

To measure the extent of DNA synthesis in bladders cultured ex vivo, organs were decompressed and incubated with 5-bromo2'-deoxyuridine (BrdU, $10 \mu \mathrm{M}$ ) for $3 \mathrm{~h}$ at $37^{\circ} \mathrm{C}$ in a $5 \% \quad \mathrm{CO}_{2} / 95 \%$ air humidified incubator. BrdU was diluted in serumfree M199 medium before addition to tissue. Following incubation, bladders were bisected transversely; one half was used for isolation of protein and the other was fixed in formalin and embedded in paraffin. Paraffin-embedded samples were sectioned and prepared for immunohistochemistry (IHC) as described above. BrdU incorporation was detected by alkaline-phosphatase-based IHC (Roche Applied Science) per manufacturer's protocol and was visualized under brightfield microscopy. A section of rat ileum served as a positive control with each set of experiments. A section incubated with secondary antibody only served as a negative control. BrdU-positive nuclei were quantified by automated counting performed by image analysis software (Image J, NIH) on 3 high power fields from 3 different sections.

The degree of apoptosis was assessed by terminal deoxynucleotidyl transferase biotin-dUTP nick end labeling (TUNEL) staining. Paraffin-embedded samples were sectioned and prepared for IHC as described above. TUNEL staining was detected by peroxidase-based IHC using the In Situ Cell Death Detection Kit (Roche Applied Science) per manufacturer's protocol and visualized under brightfield microscopy. Positive controls were created by treating bladder sections with DNAse I (Roche Applied Science) and proteinase K (Roche Applied Science) for $30 \mathrm{~min}$ at $37^{\circ} \mathrm{C}$. A section incubated with secondary antibody only served as a negative control. TUNEL-positive nuclei were quantified by automated counting performed by image analysis software (Image J, NIH) on 3 high power fields from 3 different sections.

\section{Pharmacologic Inhibition of ErbB-Dependent Signaling}

In selected experiments, we employed the EGFRspecific $^{31}$ TKI, ZD1839 (Gefitinib, Iressa, obtained from AstraZeneca, Cheshire, UK) to achieve pharmacologic blockade of EGFR-dependent signaling in bladder cells and tissue. ZD1839 was reconstituted with DMSO to a stock concentration of $20 \mathrm{mM}$. In 
preliminary experiments, NRK-52E cells were treated with $\mathrm{rEGF}(100 \mathrm{ng} / \mathrm{ml})$ in the presence of increasing doses $(0.01-50 \mu \mathrm{M})$ of ZD1839. Whole-cell lysates were subjected to SDS-PAGE and immunoblotting with antibody against phospho-EGFR (Tyr 1068, CST). Alternatively, primary bladder SMC were subjected to cyclic-stretch relaxation in the absence or presence of the indicated doses of ZD1839 or the ErbB2 inhibitor AG825. Drugs were added to cells $30 \mathrm{~min}$ before initiation of stretch, and thymidine incorporation was determined as described above. Based on these data whole bladders were treated with $10 \mu \mathrm{M}$ ZD1839 in the following ways: (1) in the media surrounding the bladder (extraluminal); or (2) in the media surrounding and the media within the lumen of the bladder (extra- and intraluminal); or (3) in the media within the lumen of the bladder (intraluminal). Specimens were subjected to sustained mechanical stretch as described above. Bladders that were not stretched and bladders that were stretched but not treated with ZD1839 served as controls for each experiment. Each time point was repeated in triplicate. Following experimental manipulation, specimens were treated with BrdU and processed for BrdU and TUNEL staining, or IHC for total and phosphorylated forms of the EGFR or the related receptor ErbB2, as described above.

\section{Statistical Analysis}

Using commercially available software (SPSS 14.0, Chicago, IL, USA), the one-way ANOVA procedure was used to test for variance among multiple comparison groups, and Tukey's test utilized for statistical significance. A $P$-value of less than 0.05 was considered significant.

\section{Results}

Previous data from our laboratory demonstrated expression of the EGFR/ErbB1 in the smooth muscle compartment of human and mouse bladder. ${ }^{22}$ To determine whether EGFR activation occurs in situ in response to bladder wall distension, we employed an ex vivo model of bladder stretch as described previously. ${ }^{3}$ This model has advantages over in vivo outlet obstruction in that systemic effects not of bladder origin are eliminated and early consequences of the distension stimulus can be monitored before gross and irreversible fibroproliferative remodeling.

Bladders were distended in vivo to $40 \mathrm{~cm} \mathrm{H}_{2} \mathrm{O}$ using water manometry and incubated ex vivo under standard cell culture conditions for $4 \mathrm{~h}, 12 \mathrm{~h}, 18 \mathrm{~h}$, and $24 \mathrm{~h}$. This pressure was selected because it is known to be a pathologically elevated urinary storage pressure associated with upper urinary tract deterioration. $^{32}$ Expression and activation status of the EGFR was determined by IHC analysis using antibodies to total and phosphorylated EGFR. At all time points studied, the EGFR was expressed in bladder SMC and was localized predominantly to the cytosol (Figure 1). Preincubation of anti-EGFR antibody with blocking peptide abolished EGFR staining, confirming the specificity of the antibody for EGFR (not shown). The level of EGFR expression did not change measurably in response to distention. However, EGFR phosphorylation was enhanced significantly at $12 \mathrm{~h}$ following initiation of stretch. EGFR phosphorylation was not detected at other time points, or in undistended specimens, suggesting that levels of phosphorylated receptor were below the limits of detection. Expression and phosphorylation of the EGFR were confirmed in whole-bladder tissue lysates by immunoprecipitation of EGFR and antiphosphotyrosine immunoblot (Figure 1). Consistent with the IHC analysis, tyrosine phosphorylation was evident only at $12 \mathrm{~h}$ of distension. Expression levels of the related RTKs, ErbB2, ErbB3, and ErbB4, were all above background as assessed by IHC, but EGFR staining intensity was strongest (not shown). ErbB2 phosphorylation was not detected under any conditions (not shown).

Next, we determined the extent of DNA synthesis and apoptosis in whole bladders by BrdU labeling and TUNEL staining, respectively. BrdU incorporation increased 23-fold in distended tissue at $18 \mathrm{~h}$ and $24 \mathrm{~h}$ compared to undistended controls $(P<0.001)$ (Figure 2). In contrast, there was a 30-fold increase in TUNEL staining at $4 \mathrm{~h}$ of stretch $(P<0.001)$, but a reduction to a $\sim 5$-fold difference between distended and undistended specimens at $24 \mathrm{~h}$ (Figure 2). These observations indicate that sustained distension results in increased apoptosis at $4 \mathrm{~h}$ and increased cell proliferation 18-24 h.

To examine the relationship between EGFR activation and cellular events triggered by the sustained stretch injury, we employed the EGFR TKI, ZD1839, to attenuate EGFR-dependent signals. To assess whether mechanical stretch-induced cell cycle transit could be affected by the EGFR inhibitor in bladder SMC, primary culture rat and human bladder SMC were subjected to cyclic stretchrelaxation in vitro in the absence or presence of ZD1839 or the ErbB2-specific inhibitor, AG825. DNA synthesis rate was determined by uptake of ${ }^{3}$ H-thymidine. ${ }^{15}$ As shown in Figure 3, stretchinduced DNA synthesis in both rat and human BSMC, consistent with previous reports. Treatment with ZD1839 significantly inhibited stretch-induced DNA synthesis at both 12 and $24 \mathrm{~h}(P<0.0001)$, whereas AG825 had no measurable effect on DNA synthesis.

To assess potential effects of the drugs in whole tissue, rat bladders were distended ex vivo in the absence or presence of $10 \mu \mathrm{M}$ ZD1839 administered: (a) intraluminally only (inside); (b) intraluminally and in the external culture medium (inside and 


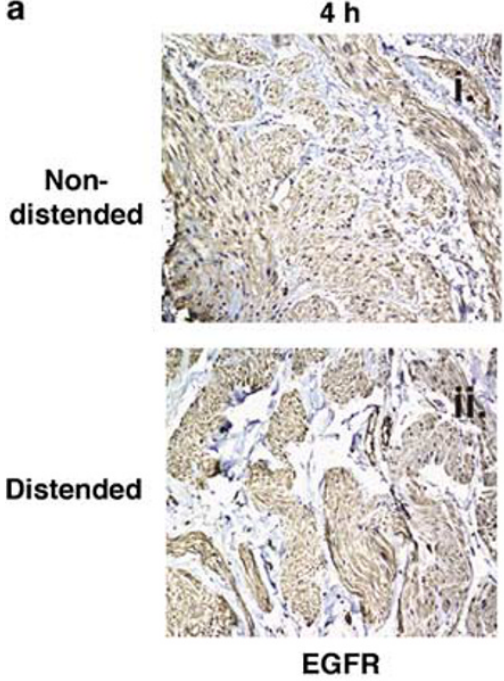

$12 \mathrm{~h}$

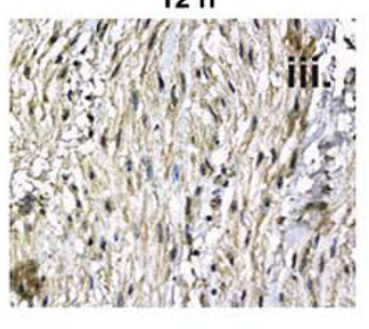

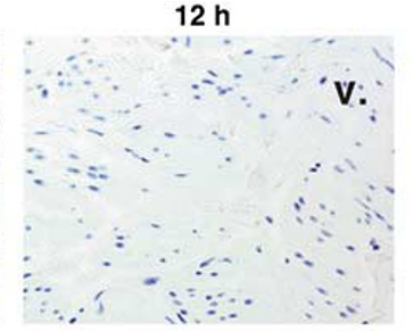

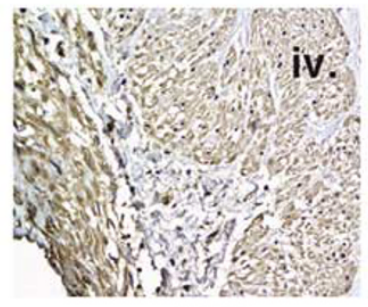

EGFR

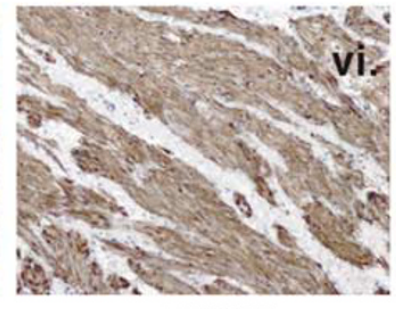

p-EGFR

b

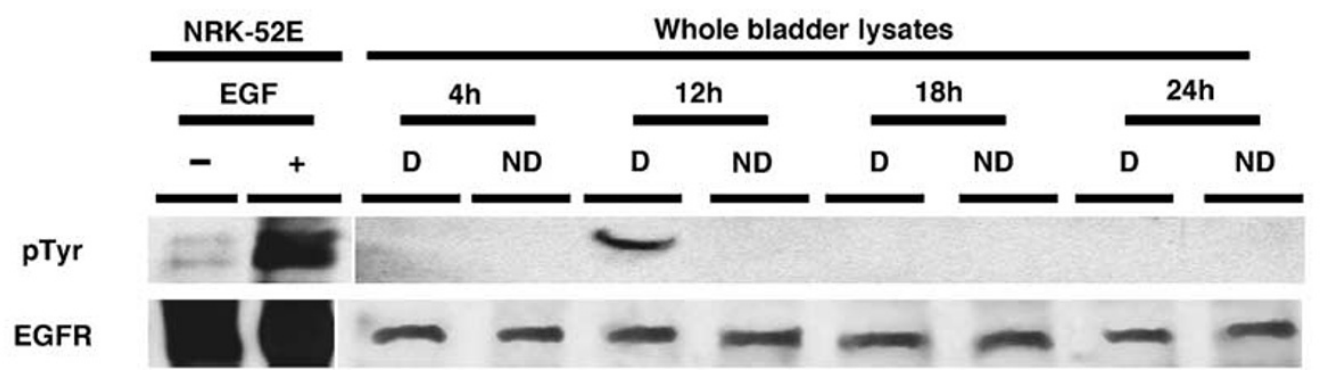

Figure 1 Distension of the bladder ex vivo promotes EGFR phosphorylation. (a) Tissue sections from whole bladders cultured ex vivo were assessed for EGFR expression and phosphorylation using IHC. Panels are from representative sections. All experiments included a secondary-Ab-only negative control in which no staining was observed in all cases (data not shown). Micrographs are shown at $\times 200$ magnification. Total EGFR expression did not vary with time or with the presence or absence of the stretch stimulus (i-iv, vii-viii). At $12 \mathrm{~h}$ of distension, but not at other time points, phosphorylation of the EGFR (p-EGFR) was detected primarily in the cytosol (v-vi). (b) IP of whole-bladder lysates $(400 \mu \mathrm{g})$ with total EGFR Ab and IB for phosphotyrosine. NRK cells treated with EGF (100 ng/ml) served as positive controls. Specificity of the EGFR Ab was confirmed with blocking peptide, which abrogated EGFR signal (data not shown). $\mathrm{D}$, distended; ND, non-distended.

outside); or (c) only in the external culture medium (outside). Regardless of the route of administration of drug, ZD1839 abrogated EGFR phosphorylation by greater than $70 \%$ (Figure 4). The decrease in staining intensity for the phosphospecific EGFR antibody was statistically significant $(P<0.01)$ for all three conditions when compared to untreated specimens (Figure 4), with no significant difference in the extent of EGFR inhibition between addition of the drug in the bladder lumen or in the external medium (not shown).

The effect of EGFR blockade on proliferation and apoptosis was determined by BrdU labeling and TUNEL staining of ZD1839-treated stretched bladder tissue. At 18 and $24 \mathrm{~h}$, intraluminal treatment with ZD1839 reduced BrdU incorporation by approximately $40 \% \quad(P<0.001) \quad$ (Figure 5), whereas TUNEL staining revealed no detectable apoptosis in response to drug treatment. These data suggest that ZD1839 attenuates distensioninduced bladder SMC proliferation by effects on cell cycle progression, as opposed to induction of cell death.

\section{Discussion}

Mechanical stretch is a well-described proliferative stimulus in diverse cell types including vascular and urinary bladder SMC, cardiac myocytes, and epithelial cells. ${ }^{1,3,4,8-12,15,23,33}$ Stretch results in the elaboration of a variety of soluble growth factors, a process that appears to be cell- and physiologic context-dependent, and the EGFR has been described as an important receptor for stretch-induced factors in various tissues. ${ }^{5-7,9-12,24-28}$ In urinary bladder SMC, stretch induces the HB-EGF gene and protein. HB-EGF is a well-known SMC mitogen $^{21,23,34}$ and ligand for the EGFR. ${ }^{22}$ The results presented here indicate that the EGFR mediates SMC growth in situ in the urinary bladder in response to distension arising from a physiologically relevant stimulus. We have also shown that pharmacologic inhibition of EGFR signaling abrogates stretch-induced SMC proliferation.

Previous studies have implicated signaling though the ErbB receptor-ligand axis in hypertrophic growth of bladder SMC exposed to 
mechanical stimuli. ${ }^{20,22,35}$ The EGFR, ErbB2, and ErbB3 are expressed in the human and murine urinary bladder, ${ }^{22}$ and stretch of bladder SMC in vitro leads to increased DNA synthesis as well as rapid activation of multiple signaling cascades. ${ }^{20,33}$ Notably, transient stretch (10 min) of bladder SMC in vitro has been reported to stimulate phosphorylation of ErbB2 but not the EGFR. ${ }^{20}$ In the present study, we observed phosphorylation only of the EGFR, but not ErbB2, following $12 \mathrm{~h}$ of sustained mechanical stretch of the intact bladder ex vivo, suggesting that signaling via these related receptors is dependent on the duration of the physiologic stimulus. We also observed a significant increase in DNA synthesis in intact specimens following $18 \mathrm{~h}$ and $24 \mathrm{~h}$ of sustained distention and in primary culture SMC following $24 \mathrm{~h}$ of cyclic stretch-relaxation in vitro. Net proliferation of bladder SMC in the ex vivo
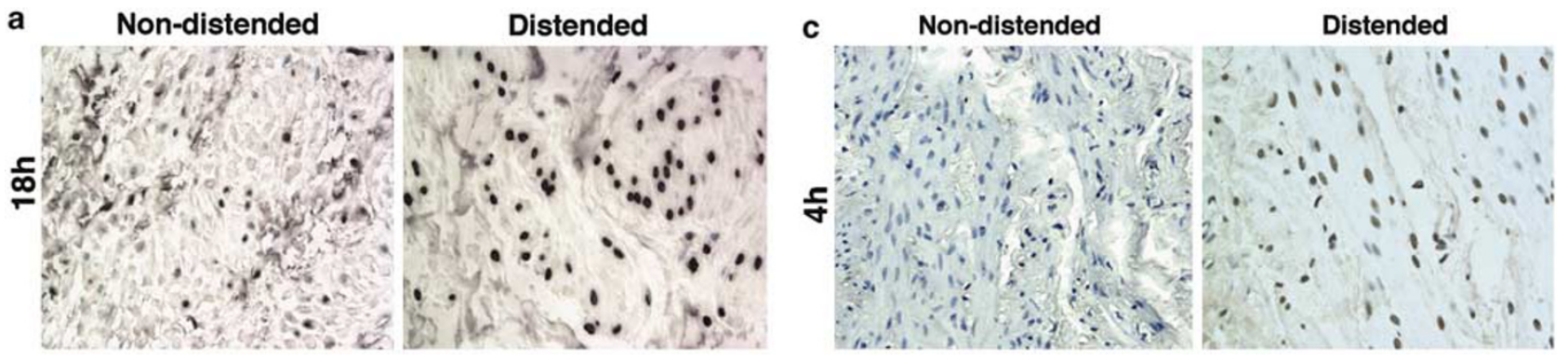

b

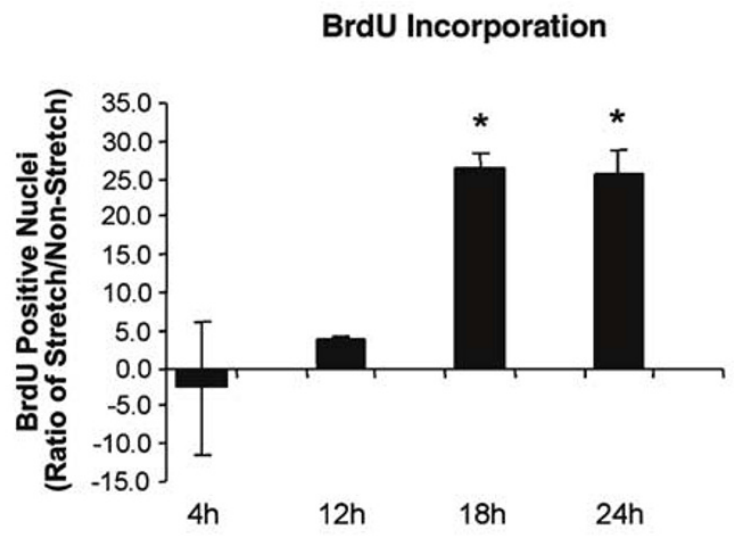

d

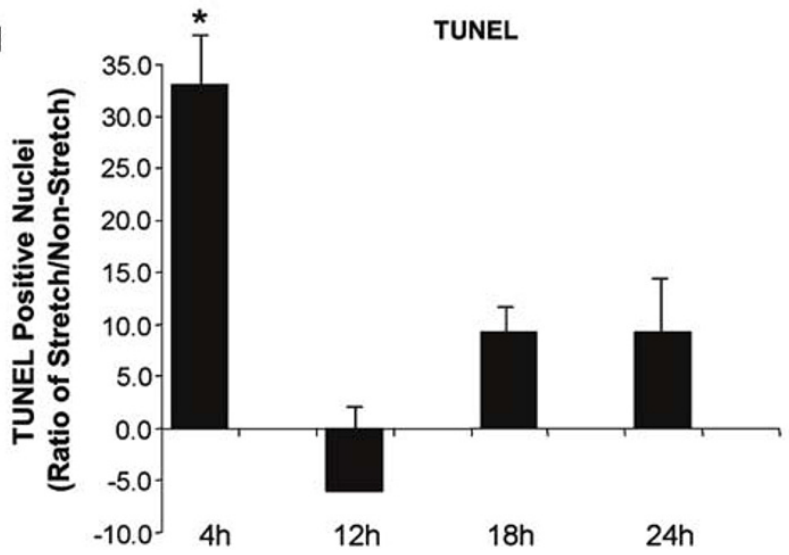

Figure 2 Sustained distension ex vivo promotes BSMC proliferation and apoptosis. (a) Tissue sections from BrdU-labeled whole bladders ( $n=3$ bladders/condition/time point) were analyzed for BrdU uptake. Representative panels are shown. Sections of ileum served as positive controls (data not shown). At $18 \mathrm{~h}$ and $24 \mathrm{~h}$, distended bladders displayed a $\sim 20$-fold increase in BrdU-positive nuclei. Magnification $\times 400$. (b) Quantitative evaluation of BrdU labeling. Data are presented as mean \pm s.d. Counting of positive nuclei was automated using image analysis software. High power fields from three sections from three different bladders were analyzed. Significant values $(P<0.05)$ are indicated $(*)$. (c) Tissue sections were analyzed for evidence of apoptosis by TUNEL staining. At $4 \mathrm{~h}$, apoptosis was maximal. Appropriate positive controls (DNase I- and proteinase K-treated sections) were used (data not shown). Magnification $\times 400$. (d) Quantitative evaluation of apoptosis as assessed by TUNEL staining. Data are presented as mean \pm s.d. Counting of nuclei was performed as described above. Significant values $(P<0.05)$ are indicated $\left({ }^{*}\right)$.

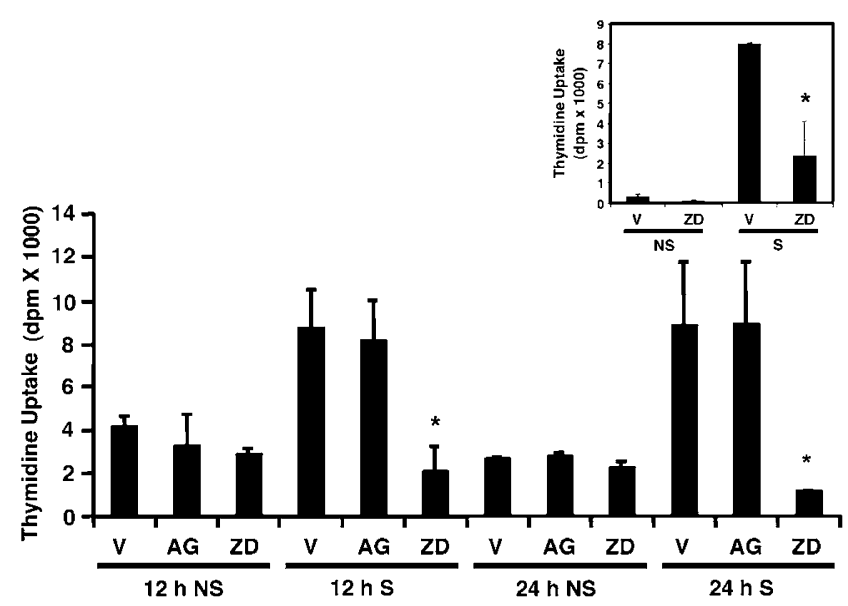

Figure 3 EGFR inhibition attenuates stretch-induced proliferation of SMC in vitro. Primary culture human BSMC were subjected to cyclic stretch-relaxation for 12 and $24 \mathrm{~h}$, in the absence or presence of either $10 \mu \mathrm{M}$ ZD1839 (ZD) or $0.35 \mu \mathrm{M}$ AG825 (AG). Cells treated with DMSO vehicle (V) served as control. $0.5 \mu \mathrm{Ci} /$ well ${ }^{3} \mathrm{H}$-thymidine was added during the final $8 \mathrm{~h}$ of stretch, and the extent of DNA synthesis was determined by measurement of ${ }^{3} \mathrm{H}$-thymidine incorporation into acid-precipitable material. Experiments were performed in triplicate, and data are presented as mean + s.d. Significant values $(P<0.05)$ are indicated $(*)$. Treatment with ZD1839 significantly inhibited stretch-induced DNA synthesis at both 12 and $24 \mathrm{~h}(P<0.001)$, whereas AG825 had no measurable effect on DNA synthesis. Inset: Primary culture rat BSMC were tested as above. Shown are data from $24 \mathrm{~h}$ stretch, demonstrating the efficacy of ZD1839 in attenuating stretch-induced DNA synthesis $(P<0.001)$. NS, nonstretched; S, stretched. 
a

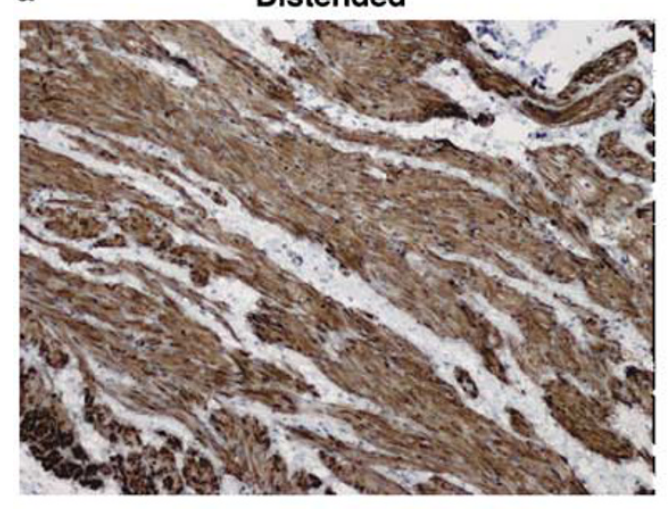

Distended + ZD1839

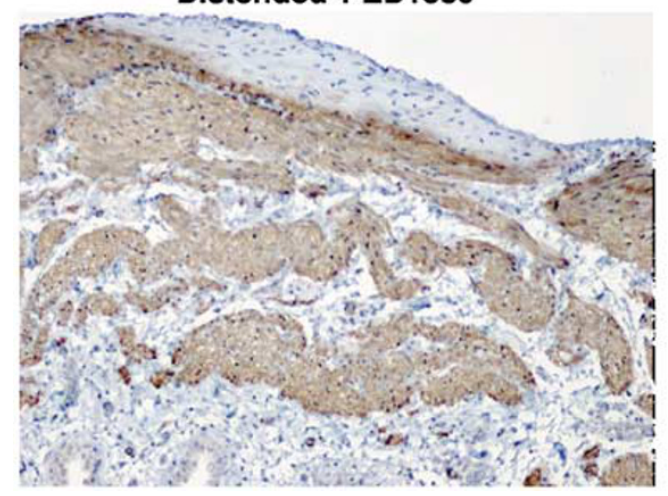

b 200 p-EGFR Intensity

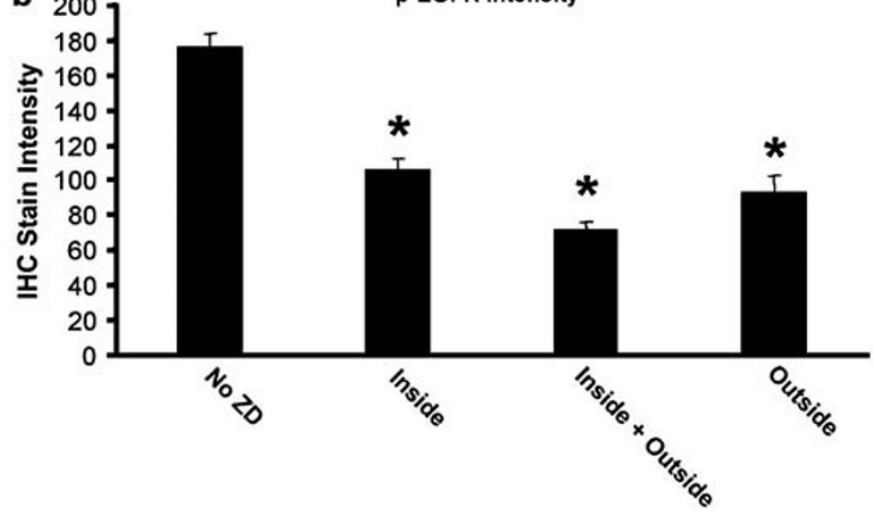

C

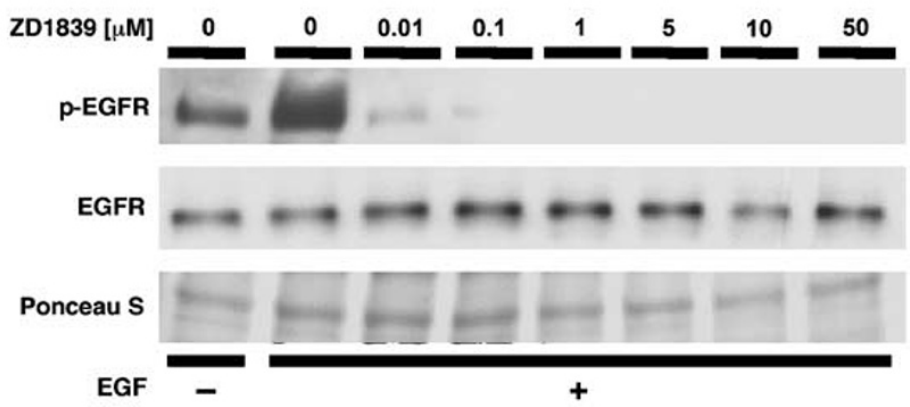

Figure 4 ZD1839 inhibits stretch-induced SMC EGFR phosphorylation ex vivo. (a) Bladders were distended ex vivo for $12 \mathrm{~h}$ in the presence or absence of ZD1839 $(10 \mu \mathrm{M})$ and EGFR phosphorylation was evaluated by IHC. The following conditions were tested: (a) No ZD1839, (b) Intraluminal ZD1839 ('Inside' in figure), (c) intraluminal + extraluminal ZD1839 (inside + outside), (d) extraluminal ZD1839 (Outside). Representative images from conditions (a) and (b), and are shown (magnification $\times 200$ ). (b) Image analysis of the intensity of p-EGFR staining under conditions (a-d). IHC staining intensity is scored in arbitrary units. The difference in p-EGFR levels between untreated bladders and those treated with ZD1839 was statistically significant $(P<0.0001)$. There was no significant difference in the level of p-EGFR between bladders receiving ZD1839 by different routes $(b-d)$. Significant values $(P<0.05)$ are demarcated with an asterisk. (c) NRK-52E cells were serum-depleted for $24 \mathrm{~h}$, preincubated for $30 \mathrm{~min}$ with ZD1839 $(0.01-50.0 \mu \mathrm{M})$, then treated with recombinant EGF $(100 \mathrm{ng} / \mathrm{ml})$ for $10 \mathrm{~min}$ on ice, $5 \mathrm{~min}$ at $37^{\circ} \mathrm{C}$. Plasma membrane fractions were isolated, resolved by SDS-PAGE, and probed for total and phosphorylated EGFR. Untreated cells, and EGF-treated cells that did not receive ZD1839 were included as controls. The phospho-EGFR signal was almost completely abolished with $0.1 \mu \mathrm{M}$ ZD1839. Ponceau S staining of the nitrocellulose membrane indicates equivalent protein loading.

model system was accompanied by a significant reduction in the rate of apoptosis by $24 \mathrm{~h}$ of distension, after increasing nearly 30 -fold above baseline at the $4 \mathrm{~h}$ time point. Our findings are in agreement with those reported by Galvin et $a l^{33}$ who demonstrated that in vitro bladder SMC apoptosis peaks following $3 \mathrm{~h}$ of stretch and is significantly diminished following $24 \mathrm{~h}$ of stretch. They concluded that mechanical stretch regulates proliferation and apoptosis in the urinary bladder and suggested that stretch may be an antiapoptotic stimulus in SMC. Our results appear to support their conclusions.

We selected the ex vivo whole-organ culture system to model sustained bladder stretch associated with outlet obstruction because it provides a means to precisely separate and isolate the tensional forces involved in bladder filling from those involved in contraction. It also maintains the complex cell matrix context of the intact organ, allows isolation of the intact organ from systemic humoral and neurological influences, and provides direct access for putative therapeutic agents. ${ }^{3}$ We have previously demonstrated that findings on stretchinduced gene expression using in vitro cell culture could be replicated in the ex vivo organ culture system. ${ }^{23}$ In addition, in the modeling of urinary bladder outlet obstruction, the sustained distension evoked in our ex vivo whole-organ culture model may represent a more accurate representation of the pathological stimulus rather than brief periods of rapid cellular deformation utilized in in vitro systems.

EGFR signaling has been studied in several $e x$ vivo organ culture models, including the cornea, ${ }^{36}$ intestinal tissue,$^{28,37}$ facial tissue ${ }^{38}$ skin, ${ }^{39}$ lung, ${ }^{40}$ and vasculature. ${ }^{5,6,24}$ In several studies, pharmacologic inhibitors have been used to modulate the EGFR signaling cascade in the whole organ. ${ }^{6,28,36,37,39}$ In this study, we utilized the EGFR TKI ZD1839 
a

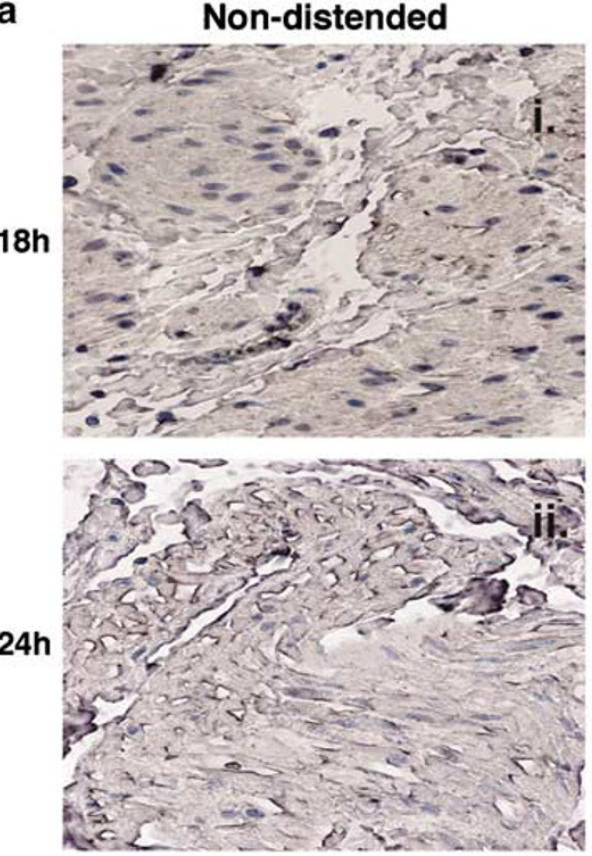

Distended
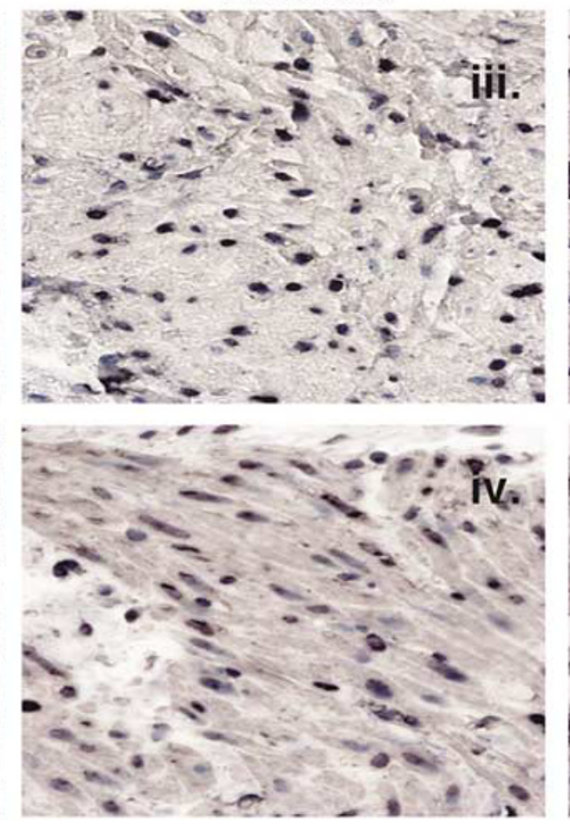

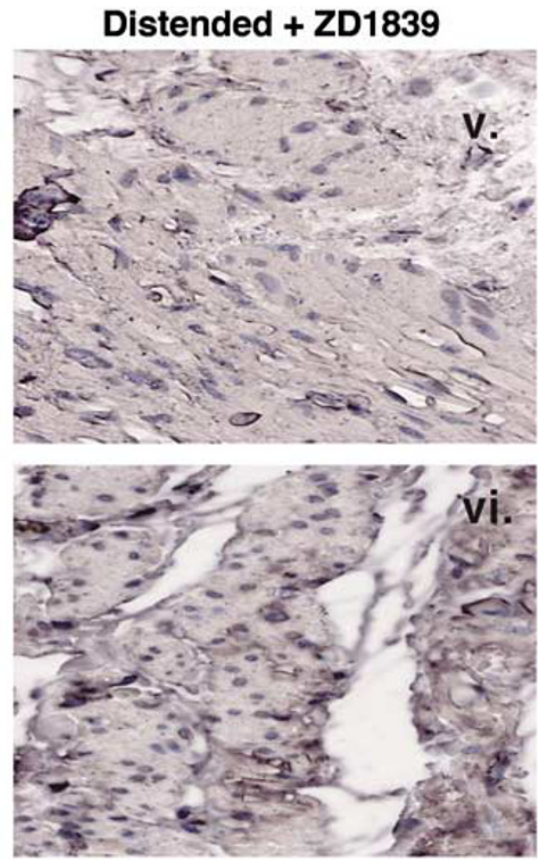

b

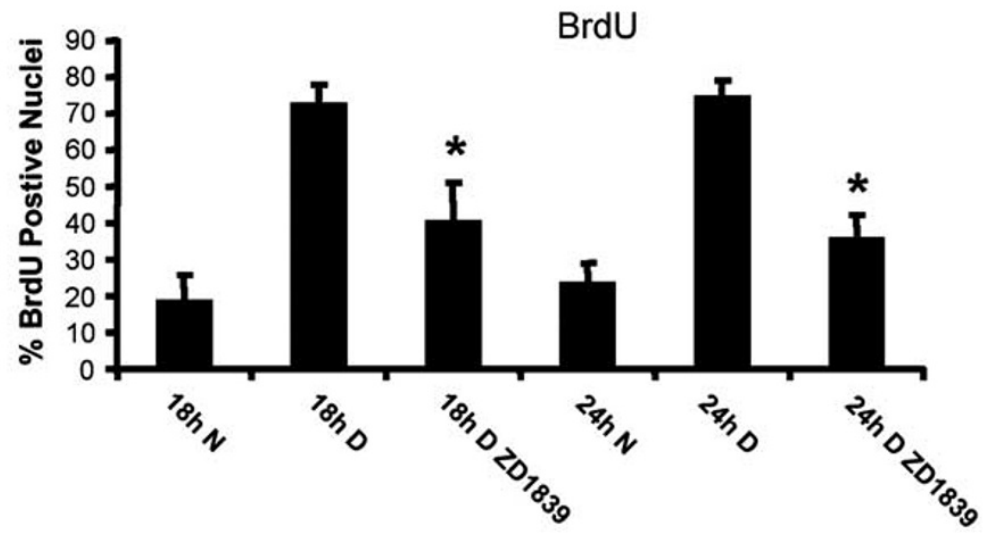

C

12h Distention
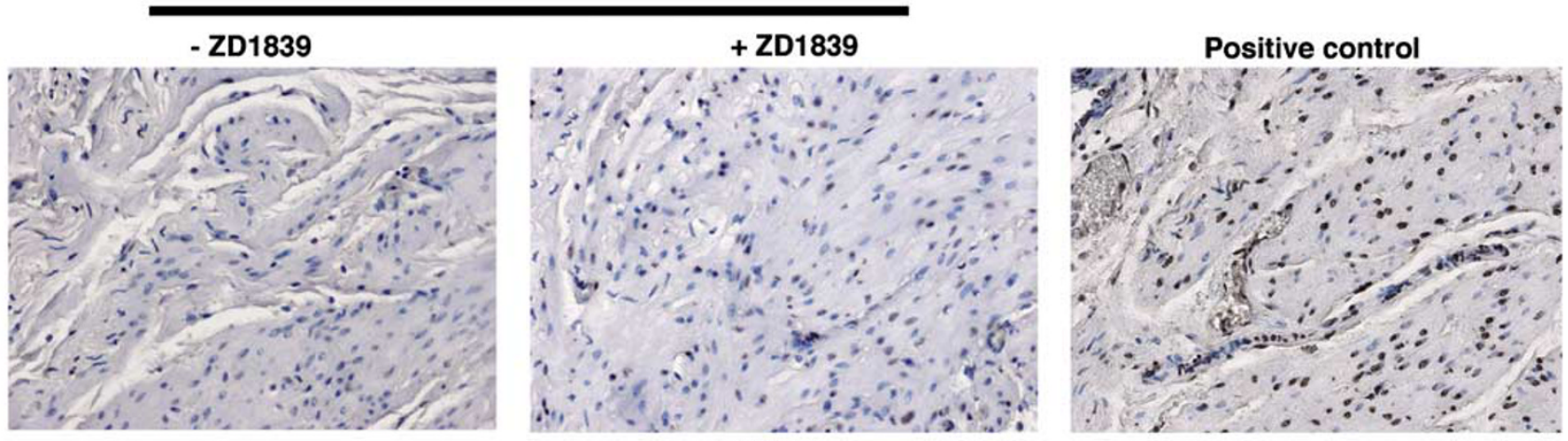

\section{TUNEL}

Figure 5 ZD1839 suppresses proliferation of bladder SMC ex vivo. (a) BrdU-labeled bladders ( $n=3 /$ condition/time point) were distended for 18 and $24 \mathrm{~h}$ in the presence or absence of ZD1839 $(10 \mu \mathrm{M})$. Panels are from representative sections. At both 18 and $24 \mathrm{~h}$ of overdistention DNA synthesis increased as is demonstrated by enhanced BrdU labeling in those sections (iii-iv). Intraluminal ZD1839 treatment reduced BrdU staining (v-vi). Magnification is $\times 400$. (b) Automated counting of BrdU-positive nuclei in these sections demonstrated significant enhancement of DNA synthesis $(P<0.001)$. Intraluminal ZD1839 treatment significantly reduced distention-induced DNA synthesis $(P<0.001)$. Values are presented as mean \pm s.d. Significant values $(P<0.05)$ are demarcated with an asterisk. $(\mathbf{c})$ Sections from bladders overdistended for 12, 18, and $24 \mathrm{~h}$ were subjected to TUNEL staining to determine the effects of ZD1839 treatment on apoptosis. A representative section at $\times 200$ magnification is shown. In the overdistended bladders treated with ZD1839, no measurable TUNEL staining was observed as compared to undistended tissue. A positive control was generated by treating bladder tissue with DNase I and proteinase K. 
(gefitinib, Iressa) to assess the relationship between distension-stimulated EGFR phosphorylation and DNA synthesis in the intact bladder. The selectivity of ZD1839 for EGFR tyrosine kinases over its closely related proteins (Erb2, Erb3, Erb4) has been previously demonstrated, ${ }^{31}$ and was a primary rationale for the study reported here. To model its potential use as an intravesical vs systemic therapeutic agent, ZD1839 was tested both as an intraluminal (intravesical) and/or extraluminal (extravesical) agent. In ZD1839-treated specimens, EGFR phosphorylation and DNA synthesis were significantly reduced and approached baseline levels. Inhibition of DNA synthesis in bladder muscle ex vivo was not associated with an increase in the apoptotic rate, suggesting that the biological effect of ZD1839 was not due simply to generalized toxicity. Interestingly, ZD1839 delivered directly into the bladder lumen was as effective at inhibiting stretch-stimulated DNA synthesis in bladder SMC as administering the drug both inside and outside the bladder. Although ZD1839 has been used most widely as systemic anticancer therapy, our findings suggest that this and other EGFR-targeted TKIs may have clinical utility for the localized, that is, intravesical, treatment of urologic disease characterized by SMC hyperplasia and hypertrophy. In addition, our results were supported by our in vitro experiments, which demonstrated that only EGFR and not ErbB2 inhibition significantly abrogated DNA synthesis following sustained mechanical stretch. The demonstration in this study that ZD1839 can interfere with EGFR-dependent signals in bladder SMC suggests that a similar strategy would be effective in modulating EGFR activation in other hollow organs that display aberrant SMC proliferation.

The inhibition of SMC proliferation in response to stretch must be examined, however, in a physiological context. Unanticipated smooth muscle growth in the bladder is usually an adaptive response to generate increased evacuative force to overcome outlet resistance, or a result of neuromuscular overdrive. In some situations (eg, outlet resistance) the growth response is compensatory, allowing the bladder to temporize urinary evacuation. Thus, illtimed antiproliferative muscle therapy, may, in fact be clinically undesirable. Ideally, early recognition or prevention of the inciting factors would prevent muscle overgrowth. Until this becomes possible, the outcome is usually a progressive and maladaptive muscle wall thickening, which often persists despite ultimate treatment of the primary insults. While our study represents one possible avenue to management of muscle overgrowth, the issue of timing of treatment and selection of which bladders are best suited and destined to respond to antiproliferative strategies will require further study.

In summary, we demonstrate (1) that a functional EGFR-dependent signaling mechanism is present in the urinary bladder, (2) that it is required for mechanical stretch-stimulated SMC proliferation, and (3) that pharmacologic inhibition of EGFR signaling abrogates stretch-induced SMC proliferation. This was demonstrated using an ex vivo whole-organ culture system, an in vitro cyclic stretch model using rodent and human BSMC, and a specific EGFR TKI in both settings. The consistency of our findings across model platforms and species suggests that the EGFR may represent a critical and modulatable component of bladder SMC response to sustained stretch injury. Further investigation in vivo may lead to a potentially novel intravesical therapeutic strategy for limiting deleterious bladder SMC hyperplasia and hypertrophy in patients with diseases characterized by chronic muscle deformation and contraction.

\section{Acknowledgements}

We wish to thank Mohini Lutchman for technical assistance and helpful discussions. This work was supported by NIH P50 DK65298, R37 DK47556, and T32 DK60442 (to MRF); the Edwin Beer Program of the New York Academy of Medicine (to RMA); the Research Grant Program of the Society for Pediatric Urology (to CRE).

\section{Duality of interest}

None declared.

\section{References}

1 Bagli DJ, Joyner BD, Mahoney SR, et al. The hyaluronic acid receptor RHAMM is induced by stretch injury of rat bladder in vivo and influences smooth muscle cell contraction in vitro (corrected). J Urol 1999;162: 832-840.

2 Deveaud CM, Macarak EJ, Kucich U, et al. Molecular analysis of collagens in bladder fibrosis. J Urol 1998; 160:1518-1527.

3 Capolicchio G, Aitken KJ, Gu JX, et al. Extracellular matrix gene responses in a novel ex vivo model of bladder stretch injury. J Urol 2001;165:2235-2240.

4 Iwasaki H, Eguchi S, Ueno H, et al. Mechanical stretch stimulates growth of vascular smooth muscle cells via epidermal growth factor receptor. Am J Physiol Heart Circ Physiol 2000;278:H521-H529.

5 Oeckler RA, Kaminski PM, Wolin MS. Stretch enhances contraction of bovine coronary arteries via an NAD(P)H oxidase-mediated activation of the extracellular signal-regulated kinase mitogen-activated protein kinase cascade. Circ Res 2003;92:23-31.

6 Zhang H, Chalothorn D, Jackson LF, et al. Transactivation of epidermal growth factor receptor mediates catecholamine-induced growth of vascular smooth muscle. Circ Res 2004;95:989-997.

7 Anderson HD, Wang F, Gardner DG. Role of the epidermal growth factor receptor in signaling strain-dependent activation of the brain natriuretic peptide gene. J Biol Chem 2004;279:9287-9297. 
8 Kudoh S, Komuro I, Hiroi Y, et al. Mechanical stretch induces hypertrophic responses in cardiac myocytes of angiotensin II type 1a receptor knockout mice. J Biol Chem 1998;273:24037-24043.

9 Alexander LD, Alagarsamy S, Douglas JG. Cyclic stretchinduced cPLA2 mediates ERK $1 / 2$ signaling in rabbit proximal tubule cells. Kidney Int 2004;65:551-563.

10 Kippenberger S, Loitsch S, Guschel $\mathrm{M}$, et al. Mechanical stretch stimulates protein kinase B/Akt phosphorylation in epidermal cells via angiotensin II type 1 receptor and epidermal growth factor receptor. J Biol Chem 2005;280:3060-3067.

11 Correa-Meyer E, Pesce L, Guerrero C, et al. Cyclic stretch activates ERK1/2 via G proteins and EGFR in alveolar epithelial cells. Am J Physiol Lung Cell Mol Physiol 2002;282:L883-L891.

12 Yano S, Komine M, Fujimoto M, et al. Mechanical stretching in vitro regulates signal transduction pathways and cellular proliferation in human epidermal keratinocytes. J Invest Dermatol 2004;122:783-790.

13 Adam RM. Recent insights into the cell biology of bladder smooth muscle. Nephron Exp Nephrol 2006;102:e1-e7.

14 Chaqour B, Han JS, Tamura I, et al. Mechanical regulation of IGF-I and IGF-binding protein gene transcription in bladder smooth muscle cells. J Cell Biochem 2002;84:264-277.

15 Park JM, Borer JG, Freeman MR, et al. Stretch activates heparin-binding EGF-like growth factor expression in bladder smooth muscle cells. Am J Physiol 1998;275: C1247-C1254.

16 Clemow DB, Steers WD, Tuttle JB. Stretch-activated signaling of nerve growth factor secretion in bladder and vascular smooth muscle cells from hypertensive and hyperactive rats. J Cell Physiol 2000;183:289-300.

17 Park JM, Adam RM, Peters CA, et al. AP-1 mediates stretch-induced expression of HB-EGF in bladder smooth muscle cells. Am J Physiol 1999;277:C294-C301.

18 Adam RM, Roth JA, Cheng HL, et al. Signaling through PI3K/Akt mediates stretch and PDGF-BB-dependent DNA synthesis in bladder smooth muscle cells. J Urol 2003;169:2388-2393.

19 Kalmes A, Daum G, Clowes AW. EGFR transactivation in the regulation of SMC function. Ann NY Acad Sci 2001;947:42-54.

20 Nguyen HT, Adam RM, Bride SH, et al. Cyclic stretch activates p38 SAPK2-, ErbB2-, and AT1-dependent signaling in bladder smooth muscle cells. Am J Physiol Cell Physiol 2000;279:C1155-C1167.

21 Freeman MR, Yoo JJ, Raab G, et al. Heparin-binding EGF-like growth factor is an autocrine growth factor for human urothelial cells and is synthesized by epithelial and smooth muscle cells in the human bladder. J Clin Invest 1997;99:1028-1036.

22 Borer JG, Park JM, Atala A, et al. Heparin-binding EGF-like growth factor expression increases selectively in bladder smooth muscle in response to lower urinary tract obstruction. Lab Invest 1999;79:1335-1345.

23 Adam RM, Eaton SH, Estrada C, et al. Mechanical stretch is a highly selective regulator of gene expression in human bladder smooth muscle cells. Physiol Genom 2004;20:36-44.

24 Merklinger SL, Jones PL, Martinez EC, et al. Epidermal growth factor receptor blockade mediates smooth muscle cell apoptosis and improves survival in rats with pulmonary hypertension. Circulation 2005;112: 423-431.
25 Yoshioka J, Prince RN, Huang H, et al. Cardiomyocyte hypertrophy and degradation of connexin43 through spatially restricted autocrine/paracrine heparinbinding EGF. Proc Natl Acad Sci USA 2005;102: 10622-10627.

26 Purdom S, Chen QM. Epidermal growth factor receptordependent and -independent pathways in hydrogen peroxide-induced mitogen-activated protein kinase activation in cardiomyocytes and heart fibroblasts. J Pharmacol Exp Ther 2005;312:1179-1186.

27 Rebsamen MC, Arrighi JF, Juge-Aubry CE, et al. Epidermal growth factor induces hypertrophic responses and Stat5 activation in rat ventricular cardiomyocytes. J Mol Cell Cardiol 2000;32:599-610.

28 Abud HE, Watson N, Heath JK. Growth of intestinal epithelium in organ culture is dependent on EGF signalling. Exp Cell Res 2005;303:252-262.

29 Kurzrock EA, Lieu DK, deGraffenried LA, et al. Rat urothelium: improved techniques for serial cultivation, expansion, freezing and reconstitution onto acellular matrix. J Urol 2005;173:281-285.

30 Orsola A, Adam RM, Peters CA, et al. The decision to undergo DNA or protein synthesis is determined by the degree of mechanical deformation in human bladder muscle cells. Urology 2002;59:779-783.

31 Brehmer D, Greff Z, Godl K, et al. Cellular targets of gefitinib. Cancer Res 2005;65:379-382.

32 McGuire EJ, Woodside JR, Borden TA. Upper urinary tract deterioration in patients with myelodysplasia and detrusor hypertonia: a followup study. J Urol 1983; 129:823-826.

33 Galvin DJ, Watson RW, Gillespie JI, et al. Mechanical stretch regulates cell survival in human bladder smooth muscle cells in vitro. Am J Physiol Renal Physiol 2002;283:F1192-F1199.

34 Wang J, Ohara N, Takekida S, et al. Comparative effects of heparin-binding epidermal growth factor-like growth factor on the growth of cultured human uterine leiomyoma cells and myometrial cells. Hum Reprod 2005;20:1456-1465.

35 Nguyen HT, Park JM, Peters CA, et al. Cell-specific activation of the HB-EGF and ErbB1 genes by stretch in primary human bladder cells. In vitro Cell Dev Biol Anim 1999;35:371-375.

$36 \mathrm{Xu} \mathrm{KP,} \mathrm{Ding} \mathrm{Y,} \mathrm{Ling} \mathrm{J,} \mathrm{et} \mathrm{al.} \mathrm{Wound-induced} \mathrm{HB-EGF}$ ectodomain shedding and EGFR activation in corneal epithelial cells. Invest Ophthalmol Vis Sci 2004;45: 813-820.

37 Duh G, Mouri N, Warburton D, et al. EGF regulates early embryonic mouse gut development in chemically defined organ culture. Pediatr Res 2000;48: 794-802.

38 Abbott BD, Buckalew AR, Leffler KE. Effects of epidermal growth factor (EGF), transforming growth factor-alpha (TGFalpha), and 2,3,7,8-tetrachlorodibenzo-p-dioxin on fusion of embryonic palates in serumfree organ culture using wild-type, EGF knockout, and TGFalpha knockout mouse strains. Birth Defects Res A Clin Mol Teratol 2005;73:447-454.

39 Varani J, Lateef H, Fay K, et al. Antagonism of epidermal growth factor receptor tyrosine kinase ameliorates the psoriatic phenotype in organ-cultured skin. Skin Pharmacol Physiol 2005;18:123-131.

40 Villanueva D, McCants D, Nielsen HC. Effects of epidermal growth factor (EGF) on the development of EGF-receptor (EGF-R) binding in fetal rabbit lung organ culture. Pediatr Pulmonol 2000;29:27-33. 